\title{
STUDIES ON THE INTERDEPENDENT EFFECTS OF STRESS AND THE ADRENAL CORTEX ON CARBOHYDRATE METABOLISM IN MAN ${ }^{1}$
}

\author{
BY THOMAS W. BURNS, ${ }^{2}$ FRANK L. ENGEL, ALBERTO VIAU, ${ }^{2}$ JAMES L. \\ SCOTT, JR., ${ }^{2}$ DOROTHY R. HOLLINGSWORTH ${ }^{2}$ AND EMILE WERK ${ }^{3}$ \\ WITH THE TECHNICAL ASSISTANCE OF EVANGELINE ROBERTSON
}

(From the Departments of Medicine and Physiology, Duke University, Durham, N. C.)

(Submitted for publication March 20, 1953; accepted April 26, 1953)

It is now well established that the adrenal cortex is involved in some fundamental manner in the metabolic response to injury and illness, but the precise mechanism by which this gland exerts its influence remains clouded. Originally, based on the observations that certain of the metabolic responses to injury do not occur in the absence of the adrenal cortex, and on the evidence of increased secretion of adrenal steroids during stress, it seemed reasonable to interpret the metabolic changes which occur after stress as direct manifestations of hypersecretion of adrenocorticotrophic hormone (ACTH) and adrenocortical steroids $(1,2,3)$. The demonstration that overdosage with ACTH or cortisone produced metabolic changes comparable to those after stress made such an interpretation even more attractive. However, as investigation in this area has been pursued, it has become increasingly apparent that the concept described above is an oversimplification and needs modification. Ingle, in a brilliant series of studies, beginning in 1943, has been chiefly responsible for demonstrating, in animals at least, that the adrenal cortex is not directly responsible for the changes under consideration, although adrenocortical hormone must be present for certain of the changes to take place $(4,5,6,7$, 8). Thus, the adrenalectomized animal exhibits a normal metabolic response to injury if maintained with an amount of adrenocortical hormone which itself does not produce overdosage effects. Under such circumstances, no increase in adrenal activity is possible, and hence adrenocortical hormone may be considered as necessary but not re-

\footnotetext{
1 Supported by a research grant from the National Institute of Arthritis and Metabolic Diseases of the $\mathrm{Na}$ tional Institutes of Health, Public Health Service.

2 Research Fellow in Medicine.

${ }^{3}$ Supported by.a Research Fellowship from the Merck Co., Rahway, N. J.
}

sponsible for certain of the observed metabolic and morphologic alterations after stress. Although the original interpretation of a direct participation of the adrenal cortex in the metabolic response to injury is still widely held, most investigators in this field, including Selye and others who were responsible for the concept, are now in agreement that the role of the adrenal is an indirect one (9, $10)$.

In this laboratory, attention has been directed for some years to the problem of the nature of the interrelationship between the adrenal cortex and stress in metabolism $(11,12,13)$. Ingle's observations have been confirmed, using different techniques. Evidence has been adduced in the rat to indicate that hypersecretion of adrenal steroids is not necessary to initiate the metabolic response to injury, although a high level of secretion may be necessary to sustain it. Thus, it was found that even large doses of ACTH, infused intravenously into the fasted intact rat, produced only delayed effects on nitrogen metabolism, whereas stressful stimuli were followed by immediate changes. It was further shown that if adrenocortical extract is administered to rats subjected to a subthreshold stress, i.e., one which induced either no or only a delayed increase in protein catabolism, the animal responds with an immediate increase in nitrogen metabolism, as if it had been subjected to a more severe stressful stimulus. These studies have led to the concept that the adrenal hormone in some manner permits the organism to respond metabolically to stressful stimuli, the magnitude of the response being dependent primarily on the stress stimulus unless an excess of adrenal hormone is present. In these terms, the so-called "overdosage effects" of exogenous adrenal steroids conceivably could be interpreted as over-responses to minor stressful stimuli, which under ordinary 
circumstances, would have no measurable impact on metabolic processes. At the moment, of course, there is no positive proof for such a concept. The present study was designed to extend these observations to one parameter of metabolism in man, namely carbohydrate tolerance. For this purpose, studies were made of the influence of cortisone acetate on the carbohydrate tolerance of normal healthy medical students as compared with ill patients in the hospital. It was found, in confirmation of the views developed from the animal studies, that subjects under the acute or chronic stress of organic illness exhibited a greater change from normal in glucose and insulin tolerance after a given dose of cortisone acetate than did healthy medical students similarly treated.

\section{MATERIALS AND METHODS}

The subjects for these studies were 143 normal medical and graduate students and 53 patients. Most of the latter group suffered from organic disease, although a few with severe neuroses were included. With the exception of the special diet control noted below, the students all ate ad libitum at the university dining halls. A dietary survey of a representative group of students indicated that the range of diet consumed by them ranged from 1900 to 3000 calories a day with from 200 to 300 grams of carbohydrate, the majority of students consuming diets containing between 2,500 and 3,000 calories. The composition of the dining hall diets was thus not notably different from the ward diet offered the patients, i.e., 2,900 calories, with 110 grams protein, 290 grams of carbohydrate and 140 grams of fat. The tolerance tests were performed on the students during the morning, after they had been sitting through the hour lecture period. Activity during the two to three hours required for the tolerance tests consisted of the walking about necessary to perform routine experiments in Physiology.

The ill subjects were selected as a cross-section of the ward population with organic diseases with the exception of four subjects with anxiety states or hysteria. The latter did not respond differently from those with organic disease and hence were included. No patient with a disease known specifically to modify carbohydrate tolerance, such as diabetes, liver disease or an endocrine disorder, was included. Patients with a family history of diabetes were excluded. Most patients were ambulatory to varying degrees and where possible were not kept strictly in bed during the tests so that their activity would be comparable to the student subjects.

The tolerance tests employed were a standard oral glucose tolerance test, using 1 gram of glucose per kilogram body weight, a glucose-insulin tolerance test modified from Fraser, Albright, and Smith (14), in which 0.1 unit of regular insulin per kilogram body weight was administered intravenously simultaneously with the glu- cose by mouth ( 1 gram per kilogram body weight), and insulin tolerance tests, using $1 / 10,1 / 40,1 / 60$, and $1 / 80$ unit of regular insulin per kilogram body weight intravenously. All subjects received 50 grams of glucose by mouth ten hours prior to the test in order to make the period of fast constant and to attempt to approximate an intake of carbohydrate of $\mathbf{3 0 0}$ grams or more on the day prior to the test. The insulin used was free of hyperglycemic factor. In order to minimize error in dosage, the insulin was diluted 1:20 with normal saline to increase the volume of the injected solution. When cortisone was given, it was taken by mouth as the crystalline suspension to assure rapid absorption four hours prior to tolerance tests in the acute experiments and every six hours around the clock in the chronic experiments. In general, the dosage used in the acute experiments was $200 \mathrm{mg}$. as a single dose and in the chronic experiments a total dose of 200 mg. per day was given, $50 \mathrm{mg}$. every six hours.

Blood was drawn at appropriate intervals into heparinized syringes, and placed in tubes containing potassium fluoride. Protein-free filtrates were prepared within a few minutes, using a copper-tungstate filtrate. Blood for glucose was analyzed by the Somogyi method (15). In the statistical analyses, comparisons were made between increments and decrements in blood sugar levels from zero time as well as between the absolute values for the points on the curves. A probability of $<.05$ was considered probably significant, one of $<.02$ or less definitely significant.

\section{Control studies}

\section{RESULTS}

To assess the effects of acute administration of cortisone acetate by mouth, three groups of ten normal subjects served as controls. One group received no hormone and the other two received by mouth 200 and $400 \mathrm{mg}$. of cortisone acetate, respectively, four hours prior to withdrawal of the initial blood samples. Blood was collected for glu-

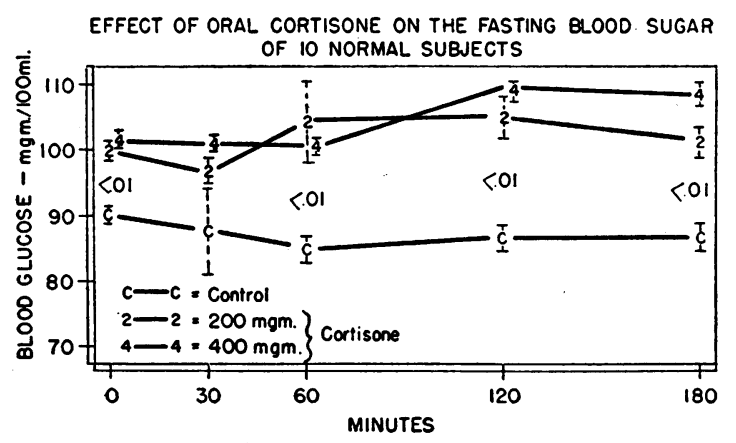

Fig. 1. Oral Ingestion of 200 or 400 mg. Of Cortisone Acetate Results in a Significant Elevation of the Fasting Blood Sugar Four Hours Later and LastING at Least Three Hours 
cose determination at $0,30,60,120$, and $180 \mathrm{~min}$ utes. Figure 1 illustrates that ingestion of cortisone was followed by a significant $(p<.01)$ and sustained increase in the fasting blood sugar during the period of four to seven hours after hormone treatment. The blood sugar levels of the untreated subjects showed a small, but statistically invalid, fall during the three hours observation, while those of the cortisone treated subjects exhibited a similarly insignificant tendency to rise. There was no significant difference between the response to 200 and to $400 \mathrm{mg}$. of cortisone.

When compared with the mean of the two pretreatment days, the fasting blood sugar. levels of seven normal subjects receiving $50 \mathrm{mg}$. of corti-

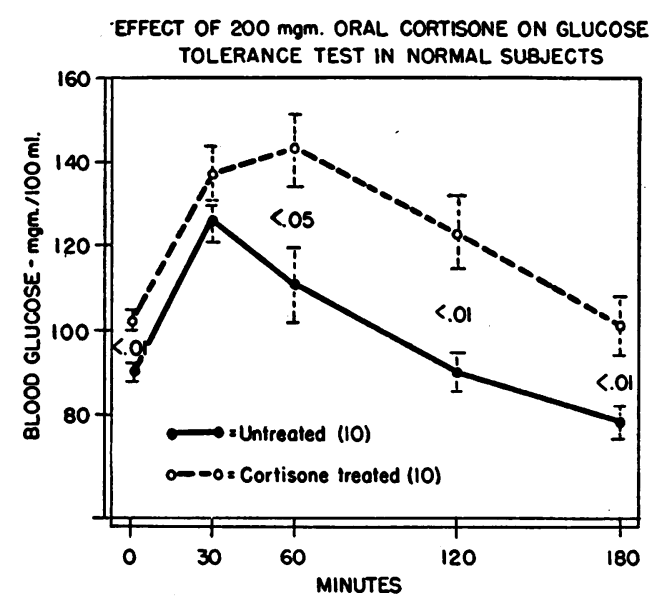

Fig. 2. Significant Alteration in the Oral Glucose Tolerance Test of Normal Subjects Four Hours after Ingestion of 200 Mg. OF Cortisone Acetate

sone acetate every six hours for eight days were significantly elevated as measured on the third and sixth day of treatment but had fallen to normal again the eighth day. The mean values and standard errors were as follows: Day -2 , pretreatment, $88 \pm 2.5$; day $0,84 \pm 4.3$ (mean pretreatment $86 \pm 1.6)$; day 3 of treatment, $96 \pm 4.1$; day 6 of treatment, $99 \pm 3.2$; day 8 of treatment, $89 \pm$ 3.9; day 1 after treatment, $83 \pm 2.1$; day 4 after treatment, $83 \pm 2.2$.

\section{Glucose tolerance tests}

Glucose tolerance tests were performed on two groups of ten subjects each, one untreated and the other pretreated with $200 \mathrm{mgm}$. of cortisone acetate orally four hours prior to the test.

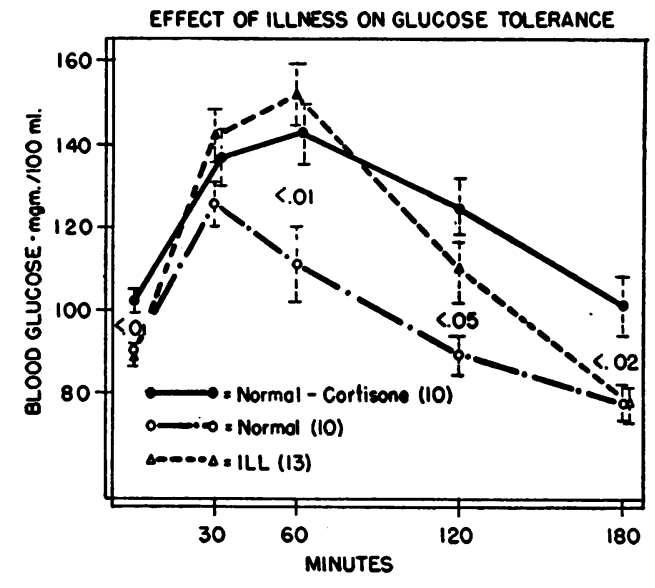

Fig. 3. Comparison of the Impairment in Glucose Tolerance of Normal Subjects Receiving 200 mg. of Cortisone with That of Ill Subjects.

Note that the 0 and 180 minute blood sugar levels are normal in the ill subjects but elevated in the cortisone treated normal individuals compared with the untreated controls.

The results exhibited in Figure 2 demonstrate clearly that blood sugar levels are significantly higher at all points in the tolerance curve of the treated subjects with the exception of the thirty minute point.

When glucose tolerance tests on thirteen ill patients were compared with those on ten untreated normal subjects and ten cortisone treated normal subjects (Figure 3 ), a clear difference between the effects of cortisone and illness is apparent. Ill subjects showed a definite impairment of glucose tolerance at the 60 and 120 minute points but not at 0,30 , and 180 minutes. This contrasts with the effect of cortisone on the healthy subjects where, as indicated in Figure 2, significant differences from normal are detectable at all times except 30 minutes after glucose.

When cortisone is administered to ill subjects, the impairment of glucose tolerance is strikingly greater than that resulting from illness alone (Figure 4) or from the administration of cortisone to healthy subjects (Figure 2). In this experiment, thirteen ill subjects had glucose tolerance tests performed before and eight of the same group after a single dose of cortisone by mouth. The data of Figures 3 and 4 demonstrate a clear difference between the response of normal and ill subjects to cortisone. Not only is the tolerance curve of the ill group receiving cortisone significantly higher 


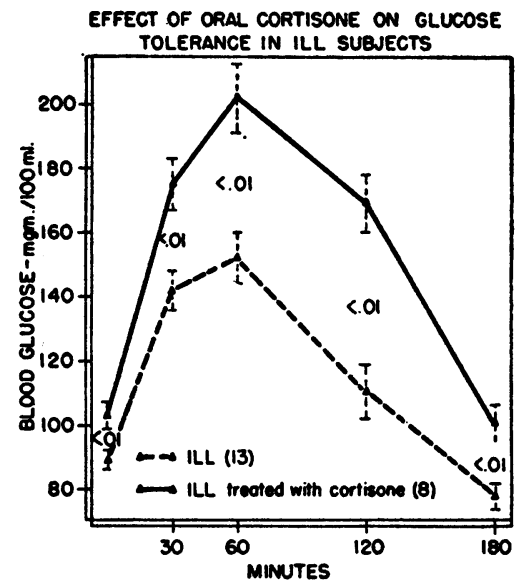

Fig. 4. Marked Impairment of Glucose Tolerance in Ill Subjects Pretreated with Cortisone Four Hours before the TEST

Compare with Figures 2 and 3.

throughout than that of the ill group not receiving cortisone, but it is also significantly higher at all but the first and last points than that of the healthy subjects receiving cortisone.

Although the patients were offered a diet adequate in calories and carbohydrate, there was no assurance or check that they consumed their entire ration. Since it is well known that impairment of carbohydrate tolerance occurs during carbohydrate restriction in the diet $(16,17)$, a control study was done to eliminate the possibility that dietary deficiency might account for the different response to cortisone noted above. Accordingly, seven normal students were given trays on the ward containing one half portions of the regular ward diet, i.e., 1,400 calories and 150 grams of carbohydrate per day. It was felt that this would correspond reasonably well with the diets consumed by the ill patients with poor appetites. At the end of seven days, a glucose tolerance test was performed on each subject, preceded by $\mathbf{5 0}$ grams of glucose the night before, as in the previous tests. Three subjects were pretreated with cortisone (200 mg., four hours before the tolerance test) and four were not. The diets were continued and at the end of the second week the tolerance tests were repeated, four subjects receiving cortisone and three not. The tolerance curves obtained were then compared with those obtained from normal subjects eating ad libitum (Figure 5). Restriction in diet alone caused slight impair- ment in glucose tolerance, apparent at $\mathbf{3 0}$ minutes $(p<.05)$. Impairment of carbohydrate tolerance after cortisone pretreatment was approximately equivalent in the group with regulated diet as compared with the group whose diet was not rigidly controlled. The curve of the diet-restricted group was not at all comparable to the curve found in the ill patients receiving cortisone (Figure 4). These results make it seem unlikely that the previous findings are explicable on the basis of dietary variations alone.

\section{Glucose-insulin tolerance tests}

In order to explore further the relationship between the effects of cortisone and illness on carbo-

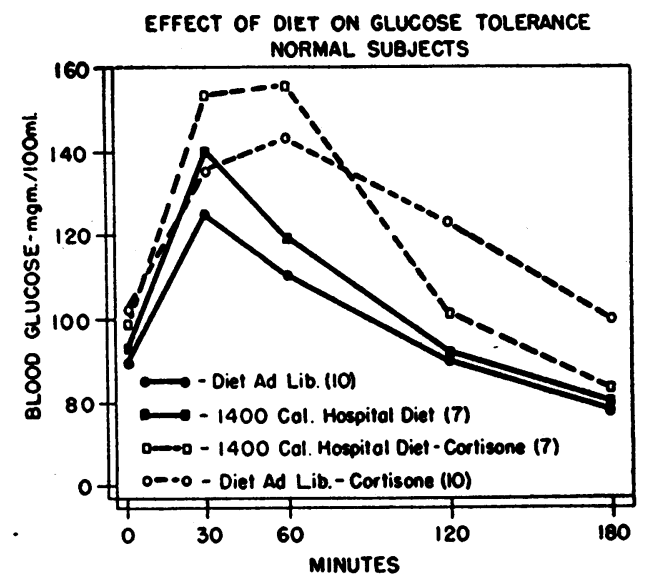

Fig. 5. Effects of Dietary Restriction on Carbohydrate Tolerance with and without Cortisone PreTREatMent in Normal SubJects

hydrate tolerance, studies comparable with those just described were done with the glucose-insulin tolerance test. This test was done in order to determine whether insulin would modify the altered response to glucose noted after cortisone pretreatment or illness.

As is apparent in Figure 6, curves A, B, and C, four hours pretreatment of normal subjects with either 200 or $400 \mathrm{mg}$. of cortisone acetate by mouth did not prevent the normal fall in blood sugar following simultaneous administration of insulin and glucose. As in previous experiments, fasting blood sugar levels were significantly higher after cortisone $(p<.01)$ and reached higher levels at two and three hours but the latter were not significant statistically. On the other hand, when the nor- 
mal subjects were treated with $200 \mathrm{mg}$. of cortisone daily for six days prior to testing, no fall in blood sugar occurred after insulin and glucose, and except for the 3 hour point, all points on the cortisone curve were significantly higher than those of the untreated controls.

When this same test was performed in ill subjects, with and without cortisone pretreatment (Figure 7), the response was strikingly different from that obtained in normal subjects, untreated or treated acutely or chronically with cortisone. No hypoglycemia developed in either of the ill groups, and significantly higher levels were achieved at 1,2, and 3 hours than in healthy subjects untreated or receiving a single dose of corti-

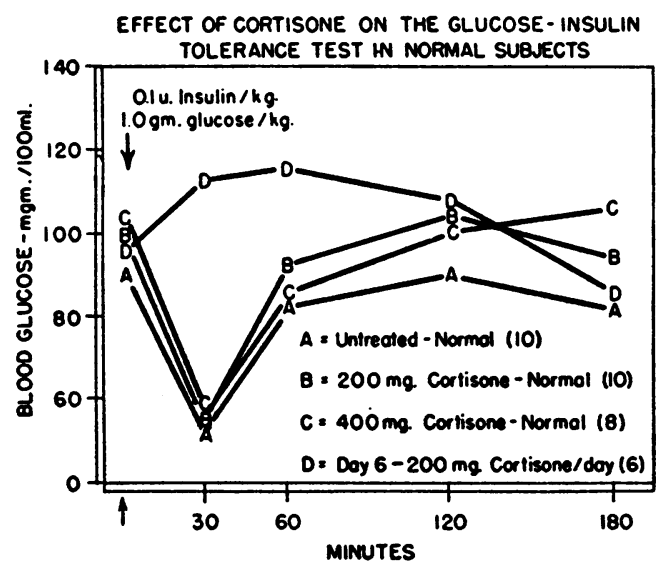

Fig. 6. Lack of Effect of 200 OR 400 Mg. OF Cortisone on the Glucose-insulin Tolerance Test Four Hours later as Compared with Impaired Tolerance on the Sixth Day of Treatment with 200 mg. of Cortisone in Normal Subjects

sone. At two and three hours, blood sugar levels in the two ill groups were significantly higher than normals on the sixth day of cortisone treatment. Although the cortisone treated ill group had blood sugar levels at all but thirty minutes higher than the untreated ill subjects, only the difference at fasting met the test of statistical significance. Samples drawn at 1, 2, and 3 hours showed a greater variation and, hence, larger standard error than in the previously described glucose tolerance tests. It is possible that if a larger group of subjects had been tested, the same differences between the two ill groups might have been significantly different, as was the case with the glucose tolerance tests.

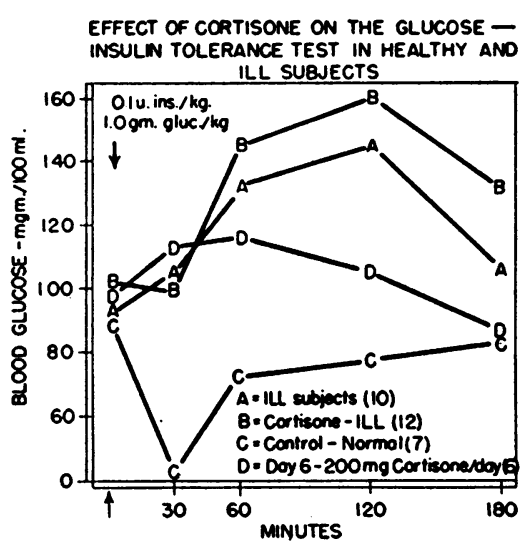

Fig. 7. Comparison of Response in Normal and Ill Subjects to Glucose and Insulin with aNd Without Acute or Chronic Cortisone Pretreatment

\section{Insulin tolerance tests}

In the past, much emphasis has been placed on the possibility that the impairment of carbohydrate tolerance, which has been reported during stress and in animals which have been treated with adrenal steroids, is due to a peripheral blocking of insulin action by adrenal steroids. This concept is supported by the experiments of Ingle in which it was found that force-fed rats which were made diabetic by the administration of very large doses of adrenal steroids were particularly resistant to insulin, requiring very large doses of this hormone to overcome glycosuria (18). The altered glucose and insulin tolerance of patients with Cushing's syndrome has also been interpreted in this fashion (14). Additional evidence that stressed individuals may exhibit insulin resistance was recently presented by Olsen and Nuetzel who reported that there was a good negative correlation between the severity of illness and the fall in blood sugar after insulin exhibited by patients during insulin tolerance tests (19). In the experiments described in the previous section, the possibility of relative insulin resistance was considered in ill patients and in normal subjects who were treated chronically with cortisone. In all these groups, no fall in blood sugar was noted after the administration of insulin and glucose in contrast to the control normal group and the normals receiving cortisone acutely. In these latter groups, there was a prompt fall in the blood sugar. The following experiments were performed in order to make quantitative measurements of the inhibi- 


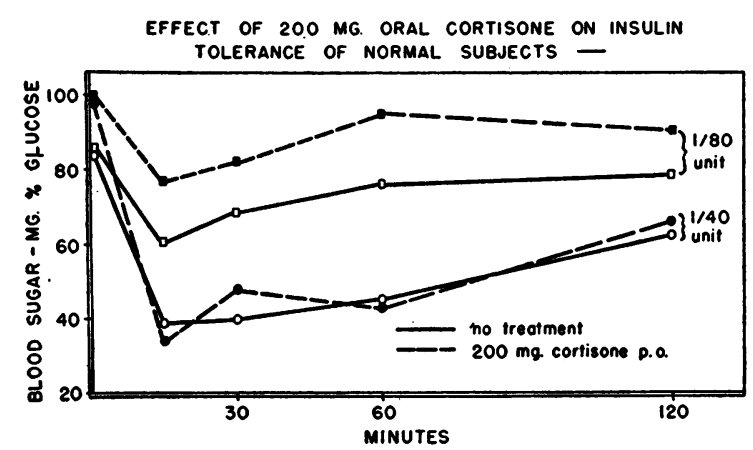

Fig. 8. Effect of 200 mg. of Cortisone on SubseQUENT Tolerance to $1 / 80$ AND $1 / 40$ OF A UNIT OF INSULIN PER Kilogram Intravenously Four Hours Later in Normal SubJects

There were ten subjects each in the $1 / 80$ unit group and six controls and four treated subjects in the $1 / 40$ unit group.

tory effects of cortisone on the response to insulin of normal and ill individuals.

For this purpose, tolerance tests were performed with four dose levels of insulin: $1 / 80,1 / 60,1 / 40$, and $1 / 10$ unit per kilogram intravenously. Figure 8 illustrates the responses of normal subjects to two dose levels of insulin, $1 / 40$ and $1 / 80$ of a unit per kilogram. In neither case, did pretreatment with cortisone modify the magnitude of the fall in blood sugar level. However, with the smallest dose of insulin all blood sugar levels were significantly higher in the cortisone treated group. This might be interpreted as evidence for some degree of insulin resistance following cortisone.

The relative ineffectiveness of cortisone in preventing the fall in blood sugar after insulin, even when the latter substance was administered in

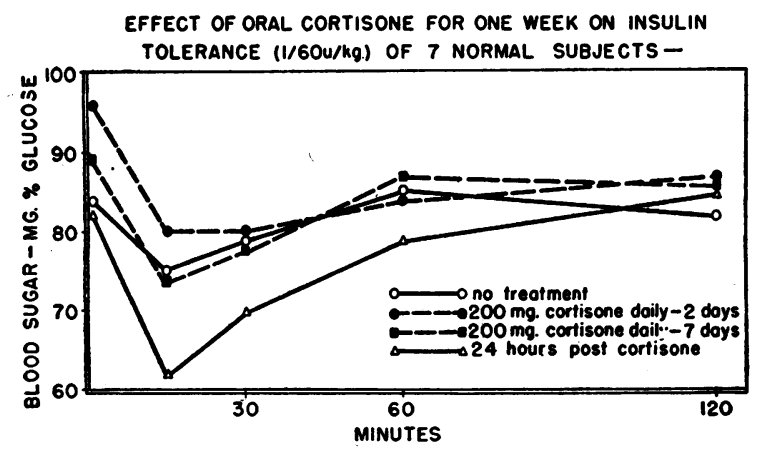

Fig. 9. Effects of Chronic Treatment with Cortisone Acetate on the Insulin Tolerance Test ( $1 / 60$ UNit per Kilogram IntravenousLy) in Normal Healthy Subjects relatively small quantities, was'surprising, especially in view of the earlier acute experiments which showed that cortisone was capable of altering the response to glucose. To assess the possibility that chronic cortisone treatment might interfere more definitely with the response to insulin in normal subjects, a group of seven subjects was treated with $50 \mathrm{mg}$. of cortisone every six hours around the clock for seven days. Figure 9 illustrates the results. Insulin tolerance tests, using $1 / 60$ of a unit per kilogram intravenously, were done the day before treatment was initiated, on the second and seventh day of treatment and 24 hours after cortisone was discontinued. Although cortisone treatment was associated with a marked alteration of the response to simultaneously ad-

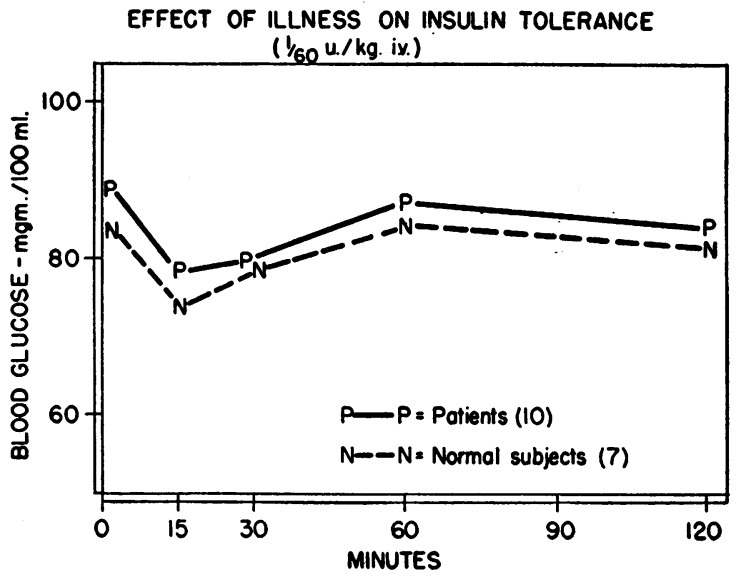

FIG. 10

ministered glucose and insulin (Figures 6 and 7) in the same subjects on the sixth day of treatment, the response to insulin alone was very little modified by cortisone treatment. The absolute fall in blood sugar was greater in the cortisone treated subjects and the level to which the blood sugar fell was the same as in the untreated group. In the tolerance tests done 24 hours after cortisone had been discontinued, during a period when one might expect relative adrenal insufficiency, the total fall in blood sugar after insulin was not significantly altered. However, the fasting level was lower and consequently the level reached after insulin was significantly lower than in the control or cortisone treated groups, a response which may be interpreted as indicating an increased sensitivity to insulin. 


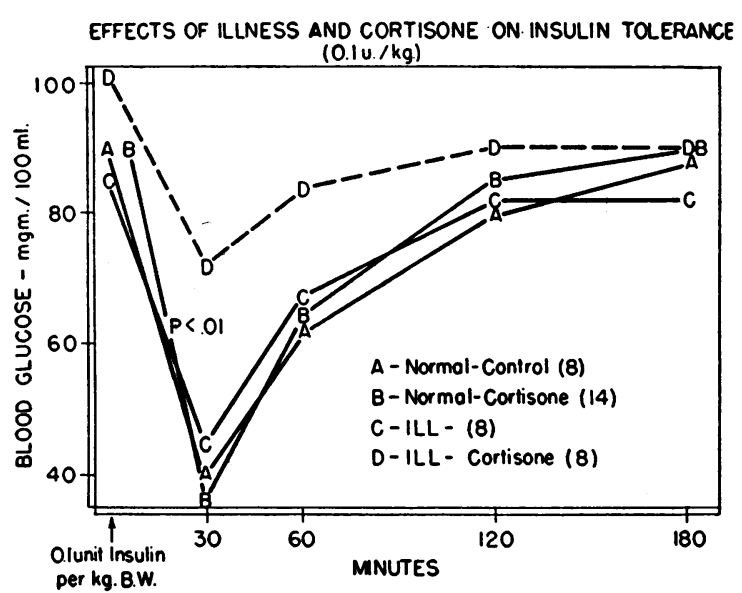

Fig. 11. Insulin Resistance (1/10 Unit PER Kilogram Intravenously) in Ill Subjects Pretreated with 200 mg. Of Cortisone Four Hours Previously as Compared with Normal Insulin Sensitivity in Untreated Ill Subjects and Normal Subjects Pretreated with CoRtisone

To determine if illness would alter the response to small doses of insulin, as has been reported (19), a group of ten patients with organic illness was tested. The dose of insulin used was $1 / 60$ unit per kilogram. The results are demonstrated in Figure 10, where the effect of illness on insulin tolerance is compared with the response of seven normal subjects. As can be seen, the response to insulin was the same in the ill group as in the untreated normal subjects. Illness, thus, was not associated with any alteration in the response to insulin under the conditions of this experiment.

It was only when cortisone treatment was superimposed on illness that any really appreciable insulin insensitivity was demonstrable (Figure 11). Insulin tolerance tests were performed on seven ill subjects, using in this instance $1 / 10$ unit per kilogram of insulin. This same group of seven patients was pretreated with $200 \mathrm{mg}$. of cortisone and the insulin tolerance test repeated in the same fashion a few days later. Hypoglycemia developed each time, but the fall noted in the subjects after cortisone was significantly less than that noted when cortisone had not been given. Thus, for the first time in this series of insulin tolerance tests, an impressive degree of insulin intolerance was noted. This result was particularly striking since it occurred in response to a comparatively large dose of insulin.

\section{DISCUSSION}

The salient features of this investigation which require comment are as follows: (1) Treatment with cortisone acetate by mouth is followed by an elevation of the fasting blood sugar within four hours which is equally significant in healthy and ill subjects. In normal subjects this is sustained for at least three hours and is not modified by doubling the dose of cortisone. (2) Chronic administration of cortisone is associated with a similar elevation of the fasting blood sugar in normal subjects, but this effect seems to wear off after about seven days. (3) In normal persons, glucose tolerance is impaired by acute cortisone treatment, but insulin tolerance is not notably modified by cortisone treatment when tested with doses of insulin ranging from $1 / 80$ to $1 / 10$ unit per kilogram body weight intravenously, even when cortisone treatment is continued for eight days. When glucose is given simultaneously with insulin to healthy individuals, no abnormality in carbohydrate tolerance is discernible after a single dose of cortisone, but a definite abnormality is apparent after six days of cortisone treatment. (4) The glucose and glucose-insulin tolerance tests yield significantly different results in ill subjects compared with healthy subjects, regardless of whether the normal subjects had been untreated or treated acutely or chronically with cortisone. The response to insulin in the ill subjects in this study, however, was not notably different from that of normal persons. (5) Pretreatment of ill subjects with a single dose of cortisone resulted in a markedly lesser blood sugar fall in the insulin tolerance test than it did in the healthy subjects. Thus, only in the cortisone treated ill subjects was any notable degree of insulin resistance noted.

The finding, that following a single oral dose of cortisone acetate there is an elevated fasting blood sugar and an impairment of glucose tolerance in healthy and ill subjects, is in keeping with well documented observations on the effects of ACTH and cortisone on carbohydrate metabolism in many subjects $(20,21,22,23)$. By itself, it contributes nothing to the understanding of the mechanism by which this response is brought about, i.e., whether by an actual impairment of glucose utilization as in pancreatic diabetes, or by an overproduction of carbohydrate from protein or possibly fat (24); 
or finally, whether the higher blood sugar levels simply reflect higher liver glycogen levels such as are known to develop in adrenal hormone treated animals (25). Forsham and Thorn (26) have favored the latter interpretation since they find serum phosphate levels to decrease to a normal degree in ACTH or cortisone treated subjects during the glucose tolerance test. The failure of the fasting sugar levels to remain elevated after seven days of cortisone ingestion is of note. It probably represents an adaptation to the metabolic effect of cortisone, perhaps through the mediation of other hormones, such as insulin.

The abnormality in glucose tolerance in ill subjects likewise is indicative of an alteration in carbohydrate metabolism well-known to occur in response to stressful stimuli $(9,27,28,29)$. Hayes and Brandt (27) have shown that despite the impaired glucose tolerance curves seen in postoperative patients, the fall in serum phosphate is normal, a response which is comparable to that reported by Forsham and Thorn in subjects receiving ACTH or cortisone (26). In our experiments, the shape of the glucose tolerance curve of ill subjects differed in one important respect from that in response to cortisone. In the former, the fasting and three hour blood sugar levels were always normal while in the latter, they were significantly elevated. This difference by itself cannot be accepted as definite evidence that the changes in carbohydrate tolerance after stress and cortisone treatment are dependent on different mechanisms, although it suggests that such might be the case.

The observation that neither illness nor acute or chronic treatment of normal subjects with cortisone modified insulin tolerance to any notable degree was unexpected. Only with the smallest dose of insulin tested, i.e., 1/80 unit per kilogram, was any degree of insulin resistance noted in healthy subjects receiving cortisone. This was apparent only in the fact that while the absolute fall in blood sugar was identical in the treated and untreated groups, the level to which the blood sugar fell in the cortisone treated group was significantly higher than in the untreated group. Since the observations of Fraser, Albright, and Smith (14) on glucose and insulin tolerance in adrenal disorders and of Ingle, Sheppard, Evans, and Kuizenga (18) on the production with ACTH or cortisone of an insulin resistant type of diabetes in the rat, it has been accepted without question that insulin insensitivity is a characteristic feature of the response to adrenal hormone overdosage. During illness, insulin resistance has also been described (19), but this depends to some extent on the severity of illness and has not been universally confirmed (30).

In the present study, a notable degree of insulin insensitivity was elicited only under certain special circumstances. Firstly, it was noted when glucose was administered simultaneously with the insulin to normal controls given cortisone for six days (but not after a single dose of cortisone) or to ill subjects, with or without a previous dose of cortisone. These experimental conditions correspond roughly to those in the report of Fraser, Albright, and Smith (14) in which insulin insensitivity in Cushing's syndrome was brought out by the glucose-insulin tolerance test, and to Ingle's work in which the amount of insulin necessary to overcome glycosuria in force-fed rats was used as the criterion of insulin resistance (18). In neither case was insulin sensitivity tested in the fasted state. Similarly, in Conn's report of diabetes in normal subjects receiving ACTH, the interpretation of insulin insensitivity was based on the occurrence of glycosuria and impaired glucose tolerance in a single subject who received protamine zinc insulin and ACTH daily, no test of insulin sensitivity being made during fasting.

The second circumstance under which insulin insensitivity was elicited directly was following a single dose of cortisone in ill subjects. This finding has special significance with regard to the interrelationship between stress and the adrenal cortex which will be discussed below. It makes the interpretation of insulin insensitivity in Cushing's syndrome or in animals receiving large doses of cortisone more complex since both these situations are associated with a high incidence of infection and other complications which superimpose a stress factor on the adrenocortical hormone overdosage effect. The results of the present experiments suggest that the mildest degrees of insulin insensitivity may only be readily apparent in the presence of a glucose load, and that in this respect the glucose-insulin tolerance may be a more sensitive indicator of insulin sensitivity than the insulin tolerance test.

Further evidence that the hypoglycemic response to insulin in the fasting state may not necessarily be greatly modified by ACTH or corti- 
sone was found in studies on two patients with panhypopituitarism. In these patients, continuous intravenous ACTH or intramuscular cortisone therapy had comparatively little effect on the blood sugar fall after insulin, but caused a marked improvement in blood sugar rise after oral glucose at 30 minutes in the insulin-glucose tolerance test $(31,32)$.

Taken as a whole, these observations in man may be considered as complementary to those previously described in animals which indicated an interdependent relationship in metabolism between stress or injury and adrenal hormone secretion. In the animal experiments of Ingle $(4,5$, $6,7,8)$ and Engel $(11,12,13)$, the adrenal factor was made a constant and the stress a variable. It was found that a constant dose of adrenal hormone enabled the adrenalectomized animals to exhibit the characteristic metabolic response to stress, although there was evidence that an increased amount of hormone might be necessary to sustain it. The present experiments were designed so that the stress (illness) and the level of adrenocortical hormone were varied independently. The data show that in the presence of the stress of illness the organism responds to an increase in circulating adrenal hormone by an impairment in carbohydrate and insulin tolerance which is out of proportion to that seen in ill subjects not treated with cortisone as well as to that exhibited by the normal, cortisone treated subject.

One cannot rule out with absolute certainty the possibility that the difference in response of the healthy and ill subjects is due to the fact that the latter are reflecting the additive effects of the ingested cortisone and a high level of endogenous adrenocortical hormone. Against this interpretation, however, are the facts that neither doubling the dose of cortisone nor chronic administration of comparatively large doses of cortisone for eight days reproduced the pattern of carbohydrate and insulin intolerance of illness, with or without cortisone treatment. As shown in the experiment with dietary restriction in normal subjects, inadequate food intake likewise cannot account for observed differences in response to cortisone.

There are, at the moment, relatively few data in the literature concerning man which give direct support to the concept derived from these studies as well as previous observations in the rat (12) that adrenal hormone overdosage may both "sen- sitize" the organism to respond metabolically to stressful stimuli as well as magnify the metabolic stress response. This is largely because few attempts have been made to isolate the variables involved in the metabolic response to stress. However, certain observations may be considered to be cogent. Thus, for example, among those who have had extensive experience with the therapeutic use of ACTH and cortisone, it is well recognized that the incidence of "hormone overdosage" effects is much greater when these substances are administered to patients who are quite ill than when they are given to subjects who have minor or localized disease, such as that involving the eye alone (33).

During the course of balance studies on patients with lymphomata receiving hydrocortisone or ACTH, Pearson and Eliel (34) observed a patient who developed an intercurrent infection. Prior to this complication, the balance of nitrogen, phosphate, potassium, sodium and chloride had become relatively stable while receiving hydrocortisone. Immediately on developing the infection, with the dose of hormone kept constant, there occurred a prompt and striking loss of nitrogen, phosphorus and potassium and a retention of sodium and chloride, with no significant increase in steroid excretion in the urine (35). Although such data must be interpreted with caution, they are consistent with the concept that in the presence of an adequate and presumably constant amount of adrenal hormone the full range of metabolic and hematologic response to stress may be exhibited.

A number of reports exist which also suggest a greater disturbance in carbohydrate metabolism after ACTH or cortisone under conditions of "stress" as compared with normals, notably in schizophrenia (36), liver disease (37) and pregnancy (38), although in none of these reports are there really adequate control studies. Appel and associates (39) described impaired insulin tolerance after four hours intravenous infusion of ACTH in patients who previously had normal insulin and glucose tolerance tests. Presumably, these were ill subjects. In our own experience, a single dose of ACTH, intramuscularly, four hours before tolerance tests, had approximately the same effect on carbohydrate tolerance in a limited number of ill subjects as did cortisone.

The results of this investigation, as well as the 
experimental work of Ingle and others, are difficult to reconcile with the concept that the metabolic abnormalities during stress and illness are direct consequences of hypersecretion of adrenal hormone. Rather, it would appear that the adrenal cortex facilitates, in some way, certain metabolic reactions which are peculiar to the tissue response to injury itself. The mechanism by which the metabolic response to injury is initiated and sustained and the precise role of the adrenal and other hormones in it, however, still remain to be elucidated.

\section{SUMMARY}

1. A series of 280 glucose, insulin and glucoseinsulin tolerance tests were performed on 150 normal subjects and 53 ill patients in order to compare the effects of cortisone on carbohydrate metabolism in the presence and absence of the stress of illness.

2. In normal subjects, a single oral dose of 200 or $400 \mathrm{mg}$. of cortisone acetate was followed by a significant elevation of the blood sugar four hours later and impairment of the glucose tolerance test. No significant changes following the same treatment could be detected in the glucose-insulin or insulin tolerance tests with doses of insulin, ranging from $1 / 60$ to $1 / 10$ of a unit per kilogram intravenously. With $1 / 80$ of a unit of insulin per kilogram, the total fall in blood sugar was not modified by cortisone treatment, but the level to which it fell was slightly higher than in the untreated group, indicating slight insulin insensitivity.

3. Chronic treatment of normal subjects with $200 \mathrm{mg}$. of cortisone daily for eight days did not modify the response to $1 / 60$ of a unit of insulin per kilogram intravenously, but did abolish the blood sugar fall previously seen when glucose was administered simultaneously with $1 / 10$ unit of insulin per kilogram intravenously.

4. Ill subjects exhibited impaired glucose and glucose-insulin tolerance tests, but normal insulin tolerance at all dose levels of insulin tested. The shapes of the curves of the impaired glucose and glucose-insulin tolerance tests in the ill subjects were significantly different from those of the normal individuals receiving cortisone.

5. Ill subjects pretreated for four hours with $200 \mathrm{mg}$. of cortisone-acetate showed significantly greater impairment of glucose tolerance and greater resistance to insulin than did normal subjects acutely or chronically treated with cortisone. Cortisone treated patients exhibited only slightly greater insulin resistance in the glucose-insulin tolerance test than did untreated patients, but markedly greater resistance than did normal subjects receiving cortisone acutely or chronically.

6. These observations are considered to be compatible with the concept that there is an interaction between stress and adrenal hormone in modifying carbohydrate metabolism. They are not reconcilable with the view that the metabolic alterations during stress are direct consequences of adrenal cortical hypersecretion.

\section{ACKNOWLEDGMENT}

We are indebted to Drs. Augustus Gibson and Elmer Alpert of the Merck Company, Rahway, New Jersey, for the Cortone and to Dr. Otto Behrens of the Eli Lilly Co., Indianapolis, Indiana, for insulin free of hyperglycemic factor used in these experiments. Thanks are also tendered to the many medical and graduate students who whole-heartedly participated in these experiments as part of a laboratory exercise in Physiology.

\section{REFERENCES}

1. Albright, F., Cushing's syndrome: Its pathological physiology. Its relationship to the adreno-genital syndrome and its connection with the problem of the reaction of the body to injurious agents ("Alarm Reaction" of Selye). The Harvey Lecture Series, 1942-43, 38, 123.

2. Browne, J. S. L., Schenker, V., and Stevenson, J. A. F., Some metabolic aspects of damage and convalescence. J. Clin. Invest., 1944, 23, 932.

3. Selye, H., The general adaptation syndrome and the diseases of adaptation. J. Clin. Endocrinol., 1946, 6, 117.

4. Ingle, D. J., Ward, E. O., and Kuizenga, M. H., The relationship of the adrenal glands to changes in urinary non-protein nitrogen following multiple fractures in the force-fed rat. Am. J. Physiol., $1947,149,510$.

5. Ingle, D. J., Some studies on the role of the adrenal cortex in organic metabolism. Ann. New York Acad. Sc., 1949, 50, 576.

6. Ingle, D. J., Parameters of metabolic problems. Rec. Prog. Hormone Res., 1951, 6, 159.

7. Ingle, D. J., The role of the adrenal cortex in homeostasis. J. Endocrinol., 1952, 8, xxiii.

8. Ingle, D. J., Some further studies on the relationship of adrenal cortical hormones to experimental diabetes. Diabetes, 1952, 1, 345.

9. Selye, H., The physiology and pathology of exposure to stress. A treatise based on the concepts of the 
general adaptation-syndrome and the diseases of adaptation. Acta Inc., Montreal, 1950.

10. Browne, J. S. L., Post-conference discussion. Proceedings of the First Clinical ACTH Conference, J. R. Mote, ed., The Blakiston Co., Philadelphia, 1950, 127.

11. Engel, F. L., Studies on the nature of the protein catabolic response to adrenal cortical extract. Accentuation by insulin hypoglycemia. Endocrinology, 1949, 45, 170.

12. Engel, F. L., A consideration of the roles of the adrenal cortex and stress in the regulation of protein metabolism. Rec. Prog. Hormone Res., 1951, $6,277$.

13. Engel, F. L., On the nature of the interdependence of the adrenal cortex, non-specific stress and nutrition in the regulation of nitrogen metabolism. Endocrinology, 1952, 50, 462.

14. Fraser, R., Albright, F., and Smith, P. H., The value of the glucose tolerance test, the insulin tolerance test and the glucose-insulin tolerance test in the diagnosis of endocrinologic disorders of glucose metabolism. J. Clin. Endocrinol., 1941, 1, 297.

15. Somogyi, M., A new reagent for the determination of sugars. J. Biol. Chem., 1945, 160, 61.

16. Goldblatt, M. W., and Ellis, R. W. B., The metabolism of carbohydrate after starvation. Biochem. J., 1932, 26, 991.

17. Conn, J. W., Interpretation of the glucose tolerance test. The necessity of a standard preparatory diet. Am. J. M. Sc., 1940, n.s., 199, 555.

18. Ingle, D. J., Sheppard, R., Evans, J. S., and Kuizenga, M. H., A comparison of adrenal steroid diabetes and pancreatic diabetes in the rat. Endocrinology, $1945,37,341$.

19. Olsen, N. S., and Nuetzel, J. A., Resistance to small doses of insulin in various clinical conditions. J. Clin. Invest., 1950, 29, 862.

20. Conn, J. W., Louis, L. H., and Wheeler, C. E., Production of temporary diabetes mellitus in man with pituitary adrenocorticotropic hormone; relation to uric acid metabolism. J. Lab. \& Clin. Med., 1948, 33, 651 .

21. Conn, J. W., Louis, L. H., and Johnston, M. W., Metabolism of uric acid, glutathione and nitrogen, and excretion of "11-oxysteroids" and 17-ketosteroids during induction of diabetes in man with pituitary adrenocorticotropic hormone. J. Lab. \& Clin. Med., 1949, 34, 255.

22. Forsham, P. H., Thorn, G. W., Prunty, F. T. G., and Hills, A. G., Clinical studies with pituitary adrenocorticotropin. J. Clin. Endocrinol., 1948, 8, 15.

23. Sprague, R. G., Power, M. H., Mason, H. L., Albert, A., Mathieson, D. R., Hench, P. S., Kendall E. C., Slocumb, C. H., and Polley, H. F., Observations on the physiologic effects of cortisone and ACTH in man. Arch. Int. Med., 1950, 85, 199.

24. Welt, I. D., Stetten, D., Jr., Ingle, D. J., and Morley, E. H., Effect of cortisone upon rates of glucose production and oxidation in the rat. J. Biol. Chem., 1952, 197, 57.

25. Long, C. N. H., Katzin, B., and Fry, E., The adrenal cortex and carbohydrate metabolism. Endocrinology, 1940, 26, 309.

26. Forsham, P. H., and Thorn, G. W., Serum phosphorus changes during glucose tolerance test. Proc. Am. Diabetes A., 1949, 9, 99.

27. Hayes, M. A., and Brandt, R. L., Carbohydrate metabolism in the immediate postoperative period. Surgery, 1952, 32, 819.

28. Eliel, L. P., Pearson, O. H., and White, F. C., Postoperative potassium deficit and metabolic alkalosis. The pathogenic significance of operative trauma and of potassium and phosphorus deprivation. $\mathrm{J}$. Clin. Invest., 1952, 31, 419.

29. Sachar, L., Walker, W., and Whittico, J., Carbohydrate tolerance, blood ketone levels and nitrogen balance after human trauma (fractures). Arch. Surg., 1950, 60, 837.

30. Mirsky, I. A., Kaplan, S. M., Podore, C. J., and Broh-Kahn, R. H., The insulin tolerance test in patients with essential hypertension. J. Clin. Invest., 1950, 29, 297.

31. Engel, F. L., and Scott, J. L., The insulin-glucose tolerance test. A modified procedure for the detection of hypoglycemia unresponsiveness in pituitary and adrenal insufficiency. J. Clin. Invest., 1950, 29, 151.

32. Burns, T. W., To be published.

33. Astwood, E. B., In discussion of Engel, F. L. (12), p. 311.

34. Pearson, O. H., and Eliel, L. P., Experimental studies with ACTH and cortisone in patients with neoplastic disease. Rec. Prog. in Hormone Res., 1951, 6, 373.

35. Pearson, O. H., Personal communication.

36. Altschule, M. D., Promisel, E., Parkhurst, B. H., and Grunebaum, H., Effects of ACTH in patients with mental disease. Arch. Neurol. \& Psychiat., 1950, 64, 641.

37. Bongiovanni, A. M., and Eisenmenger, W. J., Observations during the administration of ACTH to patients with chronic liver disease. Proceedings of the Second Clinical ACTH Conference, J. R. Mote, ed., The Blakiston Co., New York, 1951, 1, 390.

38. Jailer, J. W., Adrenal function during pregnancy and the effect of ACTH during pregnancy. Proceedings of the Second Clinical ACTH Conference, J. R. Mote, ed., The Blakiston Co., New York, 1951, 1, 77.

39. Appel, S. B., Gluck, J. L., Reichman, S., Springer, C., Schlecker, A. A., Miller, A., Goldman, A. M., and Kupperman, H. S., The effect of adrenocorticotropic hormone on blood glucose and insulin hypoglycemia. J. Clin. Endocrinol. \& Metab., 1952, 12, 979. 